\title{
Convergencia de medios. Plataformas audiovisuales por Internet (Over-The- Top) y su impacto en el mercado audiovisual en España
}

\section{The convergence of media. Internet audiovisual platforms (Over- The-Top) and their impact on the audiovisual market in Spain}

José Borja Arjona Martín. Universidad de Granada. España.

jbarjona@ugr.es

[CV] $\mathrm{C}$

Investigación financiada a través del proyecto $I+D+i$ : Nuevos consumos frente a viejos estereotipos: Análisis de la recepción por parte de la juventud española de sus representaciones televisivas actuales (CSO2017-85483-R). Programa Estatal de Investigación, Desarrollo e Innovación Orientada a los Retos de la Sociedad, en el marco del Plan Estatal de Investigación Científica y Técnica y de Innovación (convocatoria 2017), Ministerio de Economía, Industria y Competitividad.

Cómo citar este artículo / Referencia normalizada

Arjona Martín, J. B. (2021). Convergencia de medios. Plataformas audiovisuales por Internet (Over-The-Top) y su impacto en el mercado audiovisual en España. Revista Latina de Comunicación Social, 79, 35-52. https://www.doi.org/10.4185/RLCS-2021-1496

\section{RESUMEN}

Introducción. De entre la oferta actual de televisión de pago destaca el impacto de los operadores OTT (servicios audiovisuales en streaming) en la transformación del panorama actual del mercado audiovisual español. Objetivos e Hipótesis. El objetivo general consiste en analizar la situación actual del panorama televisivo en España y compararlo con los datos disponibles acerca de la irrupción de los operadores OTT. Partimos de la hipótesis que plantea una situación inicial de crecimiento hacia un modelo saturado pero de gran alcance, lo que permite fragmentar las audiencias aún más pero que pone en discusión el modelo lineal televisivo y plantea serias cuestiones acerca de la viabilidad de su modelo de negocio. Método. Se llevó a cabo una revisión exhaustiva de la información publicada por fuentes secundarias, bien periódicas o no, de ámbito nacional, así como de prensa especializada. Resultados. Se confirma el descenso del consumo de televisión lineal en beneficio de la IPTV y los OTTs. En España los ingresos de la televisión de pago se equiparan a los de la televisión en abierto, confirmando el cambio de modelo hacia un sistema de televisión basado en el vídeo bajo demanda y los dispositivos móviles. El mercado de los OTT es cada vez más diverso pero se encuentra muy concentrado en sólo dos operadores, Netflix y HBO España. Conclusiones. Se confirma la hipótesis al encontrar un panorama audiovisual saturado que supone un beneficio para los espectadores en tanto al incremento de la calidad de las producciones, especialmente las relacionadas con la ficción, pero 
que conlleva también un problema de visibilidad de los contenidos y un problema de agotamiento para los espectadores.

PALABRAS ClAVE: Over-The-Top; Televisión; Convergencia; Mercado Audiovisual; Distribución online.

\begin{abstract}
Introduction. Among the current pay television offer, the impact of OTT operators (streaming audiovisual services) in the transformation of the current panorama of the Spanish audiovisual market stands out. Objectives and Hypotheses. The general objective is to analyze the current situation of the television panorama in Spain and compare it with the available data about the emergence of OTT operators. We start from the hypothesis that it raises an initial growth situation towards a saturated but powerful model, which makes it possible to fragment audiences even more, but which calls into question the linear television model and raises serious questions about the viability of its business model. Method. An exhaustive review of the information published by secondary sources, whether periodic or not, at the national level, as well as of the specialized press was carried out. Results. The decrease in linear television consumption in benefit of IPTV and OTTs is confirmed. In Spain, pay television revenues are equal to those of free-to-air television, confirming the change of model towards a television system based on video-on-demand and mobile devices. The OTT market is increasingly diverse but is highly concentrated in just two operators, Netflix and HBO Spain. Conclusions. The hypothesis is confirmed by finding a saturated audiovisual panorama that represents a benefit for viewers in terms of increasing the quality of productions, especially those related to fiction, but which also entails a problem of visibility of the contents and a problem of exhaustion for the spectators.
\end{abstract}

KEYWORDS: Over-The-Top; Television; Convergence; Audiovisual Market; Online distribution.

\title{
CONTENIDO
}

1. Introducción 2. Objetivos e hipótesis 3. Metodología 4. Definición de Over-The-Top Player 5. Resultados 7. Conclusiones 8. Bibliografía

\section{Introducción}

Al igual que otras industrias culturales, la televisiva, tendente a la concentración empresarial y a la integración tanto vertical, como horizontal, confirma en España un escenario duopólico marcado por la división de las audiencias en pequeñas porciones más o menos segmentadas, en un contexto en el que se confirma la progresiva evolución positiva de la televisión de pago a manos de las compañías de telecomunicaciones y de los emergentes operadores Over-The-Top. Sin embargo, la industria televisiva española mantiene aún cierta fortaleza en el consumo de televisión de flujo o lineal en abierto, pese a que los ingresos por publicidad se hayan frenado en el año 2019, como veremos más adelante.

Desde que se hicieran efectivas las absorciones de Cuatro por Telecinco y La Sexta por Antena 3 desde 2009 y 2011, conformando los grupos Mediaset España y Atresmedia, respectivamente, su control sobre las audiencias y las inversiones publicitarias ha sido prácticamente absoluta. Se confirma que para la televisión en abierto no existe otro modelo de negocio viable que no sea sino el de la concentración (García Santamaría, Pérez Serrano y Alcolea Díaz, 2014: 398). Según los últimos datos publicados por InfoAdex relativos a los ingresos por publicidad para el año 2019, Mediaset y Atresmedia concentran el $84,1 \%$ de la cuota de mercado, lo que corresponde a unos ingresos de 869 millones de euros para Mediaset, suponiendo un descenso del -5,7\% respecto a 2018, y de 815 millones para Atresmedia, que registra un $-5,5 \%$ menos que en el anterior ejercicio. Según este estudio la 
televisión convencional pierde un 5,8\% de inversión publicitaria respecto a 2018, alcanzando la cifra total de 2.002,8 millones de euros (InfoAdex, 2020).

Todo ello cuando la suma de las audiencias (share o cuota de pantalla) de las tres cadenas generalistas de nivel nacional (La1, Antena 3 y Telecinco) ha registrado en febrero y marzo 2020 mínimos históricos - 35,8 y 35,9\%, respectivamente - en beneficio de otras cadenas (temáticas) y otros medios de distribución de contenidos audiovisuales, como los operadores IPTV y OTT (Barlovento Comunicación, 2020). No obstante, pese a estos descensos en las audiencias, Mediaset y Atresmedia han conseguido, progresivamente, acaparar cada vez mayores inversiones publicitarias desde que iniciaran sus procesos de concentración.

A pesar de la recuperación de las inversiones en publicidad durante el trienio 2014-2016, periodo en el que se recuperaron casi 500 millones de euros respecto a 2007 (año en el que se registró una meta histórica de cerca de 3.500 millones de euros), este crecimiento ha sufrido una desaceleración desde 2017.

Como se analizará a continuación, los ingresos de la televisión en abierto y la televisión de pago prácticamente han llegado a equipararse en estos últimos años. Por consiguiente, la televisión de pago es el sector que está consiguiendo consolidar su crecimiento año tras año, suponiendo ya un 25,7\% del total del consumo televisivo en 2019 (Barlovento Comunicación, 2019).

De entre la oferta actual de televisión de pago destaca el impacto de los operadores OTT (servicios audiovisuales en streaming) en la transformación del panorama actual del mercado audiovisual español. Su crecimiento es exponencial, duplicando en algunos casos sus suscriptores en tan sólo seis meses (como es el caso de Netflix, por ejemplo). Actualmente encontramos multitud de OTT operando en nuestro mercado, entre ellos, Disney+, AppleTV+, Rakuten Wuaki, Filmin, Amazon Prime Video, Netflix, HBO España, Sky o FlixOlé, esta última dedicada principalmente al cine español.

Estas plataformas acrecientan, aún más si cabe, la sobreabundancia de la oferta audiovisual en el mercado español a través de los diversos medios de transmisión existentes (ondas hertzianas, cable, satélite e internet). A la luz de los procesos de concentración ocurridos en la televisión en abierto y en base al exponencial crecimiento de la IPTV y los operadores OTT son muchas las preocupaciones y las preguntas que se plantean tanto las compañías tradicionales, como los consumidores. Parece que estamos asistiendo en los últimos años a una burbuja en los servicios audiovisuales de las OTT y, por ende, en la oferta audiovisual disponible. John Landgraf hablaba allá por 2015 de la Peak TV era para referirse a una época de sobreabundancia televisiva (Marcos, 2016). Esta sobreabundancia es signo de un cambio de paradigma, caracterizado por un aumento de plataformas y dispositivos y una aún mayor fragmentación de los espectadores. Sin embargo, ante esta nueva edad de oro, especialmente en la ficción, nos planteamos si estos modelos de producción y negocio, planteados por las OTT, serán viables a largo plazo o bien se moderarán una vez la selección natural haya hecho su trabajo y las compañías finalmente tomen posiciones en el mercado.

\section{Objetivos e hipótesis}

En base a las cuestiones recogidas previamente y a los desafíos que deben afrontar tanto los viejos, como los nuevos operadores, uno de los primeros objetivos de este estudio consiste en ponderar los límites definitorios del propio concepto Over-TheTop para referirse a la distribución de contenidos audiovisuales. A partir de este punto, realizaremos un análisis de la situación actual del panorama televisivo en España y su comparación con los datos disponibles acerca de la irrupción y desarrollo de los operadores OTT en nuestro mercado. 
A raíz de estos objetivos, planteamos una hipótesis de partida a partir de la observación de los numerosos movimientos de diversas compañías por posicionarse en el prometedor mercado de la televisión y el vídeo por internet (OTT). Las suscripciones y los ingresos crecen a gran velocidad y en espacios de tiempo relativamente breves, por lo que los principales operadores tratan de definirse en un mercado cada vez más saturado. Prevemos un proceso de selección natural que determinará qué compañías finalmente dominarán el mercado de los contenidos distribuidos por la red pública de internet en forma de oligopolio, en el que tendrán, asimismo, un peso fundamental los servicios adicionales de OTT de compañías de telecomunicación (Movistar, Vodafone y Orange). Por lo que observamos una situación inicial de crecimiento hacia un modelo saturado pero de gran alcance, lo que permite fragmentar las audiencias aún más que pone en discusión el modelo lineal televisivo —al menos en lo que a televisión de pago se refiere - y plantea serias cuestiones acerca de la viabilidad de su modelo de negocio.

\section{Metodología}

Con el objeto de cumplir con estos objetivos y dar respuesta a la hipótesis planteada se ha llevado a cabo una revisión exhaustiva y crítica de la información publicada por fuentes secundarias de ámbito nacional. Igualmente, los datos aportados por estas fuentes son evaluadas y contextualizadas a la luz de la información recogida por la prensa especializada y la literatura académica.

Por tanto, este artículo presenta un estudio del escenario audiovisual español mediante el análisis de información proporcionada por fuentes como InfoAdex, la Comisión Nacional del Mercado y la Competencia (CNMN), Barlovento Comunicación, el informe Televidente 2.0 -elaborado por Telefónica, Ymedia y The Cocktail Analysis- , el Observatorio Europeo del Audiovisual y prensa especializada, haciendo especial hincapié en el cada vez más importante papel que los Over-The-Top Players están desempeñando en el panorama audiovisual español. De este modo, el presente análisis puede proporcionar una suerte de radiografía específica que ayude a avanzar en el conocimiento de los efectos de la digitalización y la convergencia mediática en la producción y consumo audiovisuales.

\section{Definición de Over-The-Top Player (OTT)}

Los límites que definiría a un operador Over-The-Top pueden ser bastante difusos. Todavía más cuando las compañías tradicionales de televisión de pago, como HBO, o las propias cadenas de televisión comenzaron a lanzar sus propios servicios OTT. Pero es que el concepto Over-The-Top también es utilizado para referirse a otros servicios que utilizan la red pública de Internet y que nada tienen que ver con la industria audiovisual. El European Audiovisual Observatory (2016: 24) recoge la definición que el Body of European Regulators of Electronic Communications (BEREC) hace de los servicios Over-The-Top, y de la que se deduce que un OTT podría definir a cualquier proveedor de contenidos, de servicios de comunicación u otras aplicaciones. La nota en común de todos ellos sería su desvinculación de los proveedores de acceso a Internet. Por tanto, ni sus servicios, ni sus contenidos, poseen un control de distribución al usuario, lo que podría afectar a su calidad final. De ahí que BEREC equipare los OTTs a cualquier otro servicio de comunicaciones electrónicas. Por lo que Skype, WhastApp o Spotify, entre otros muchos, también serían considerados OTTs.

Según el observatorio europeo, desde el año 2010, se ha venido empleando este término para hacer referencia a servicios generales de acceso online a contenidos audiovisuales bajo demanda. Sin embargo, también existen otros usos más restrictivos que hacen referencia a aplicaciones concretas para televisiones conectadas, smartphones o tablets, entre otros dispositivos. 
Por otra parte, Simpson y Greenfield (2009), sin utilizar esta denominación específicamente, proporcionaron una de las clasificaciones más completas de las diferentes modalidades de distribución de contenidos audiovisuales a través de redes IP (Internet Protocol). Inicialmente, estos autores distinguieron entre dos términos que parecieron suficientes para definir la panorámica de los servicios audiovisuales por Internet: IPTV e Internet Video. Con el primero trataron de describir a los servicios de televisión lineal por suscripción que las compañías de telecomunicaciones empezaron a comercializar con la generalización de la banda ancha. Con el segundo, básicamente, distinguían al resto de propuestas de distribución de vídeo por Internet.

Sus diferencias parecían claras y suficientes para determinar las características de este mercado. Sin embargo, en un contexto de rápidos cambios tecnológicos, se hizo necesario delimitar o elaborar nuevos conceptos que pudieran designar con mayor precisión otros usos y contenidos más recientes.

Con el objeto de completar el ámbito de los servicios audiovisuales por Internet, Simpson y Greenfield propusieron la creación de dos nuevas categorías situadas entre IPTV e Internet Video. A estas categorías las denominaron Internet TV e IPVOD.

El primer término, Internet TV, es similar en algunos aspectos a Internet Video, pero muestra una diferencia fundamental: con esta categoría se define a los servicios de canales de contenido en streaming continuo (emisión lineal) en lugar de acceder a un catálogo de archivos de vídeo que puedan ser reproducidos bajo demanda. IPVOD, por su parte, delimita a la producción profesional de vídeo que puede ser reproducida bajo demanda. En algunos casos es habitual el uso de fuertes restricciones de los derechos de uso (DRM).

Asimismo, los servicios de vídeo por IP pueden clasificarse en dos categorías según el tipo de red de distribución que emplean. Básicamente si utilizan redes públicas o redes privadas. La red pública de mayor relevancia es Internet, donde los usuarios de cualquier punto del mundo con conexión pueden acceder a una basta relación de fuentes con contenido videográfico (si las restricciones por región lo permiten). Si bien estas fuentes pueden tener URLs públicas o privadas, todas ellas comparten la misma red de distribución, es decir, internet.

Los tres modelos de distribución sobre red pública (IPVOD, Internet TV e Internet Video) deben ser capaces de funcionar sobre entornos best-effort (esfuerzo óptimo), en los que proveedores y receptores deben adaptarse a la pérdida de paquetes de datos y posibles retrasos por el tráfico de la red.

IPTV es la única modalidad que emplea redes privadas, de acuerdo a la clasificación elaborada por Simpson y Greenfield. Cada usuario está conectado directamente a una red de acceso restringido, con lo que se proporciona un mayor control sobre los parámetros que afectan a la manera en que el vídeo será envíado hasta el cliente.

Los servicios de IPTV ofrecen cientos de canales de programación continua, previo pago de una suscripción general o específica (paquetes temáticos). Por su parte, el número de canales sobre Internet TV está sujeto a variaciones impredecibles y volátiles. Muchos de los canales disponibles en Internet TV existen en otros medios convencionales. Sin embargo, en algunos casos, los canales en Internet TV son reemplazados por portales de vídeo (Internet Video) que multiplican la flexibilidad de uso por parte de sus usuarios.

Los servicios de IPTV ofrecen una experiencia de usuario similar a cualquier servicio de televisión digital por cable o satélite. De hecho se ha erigido como la fórmula de televisión de pago más importante en España, incrementando su cuota de mercado año a año. 
En cuanto al resto de categorías, los usuarios de un sistema IPVOD dispondrán de una experiencia semejante a los servicios de vídeo bajo demanda de televisión por cable o al funcionamiento de un vídeo grabador digital (DVR). Internet TV e Internet Video proporcionaban tradicionalmente una experiencia parecida a la navegación web, debido en ambos casos al dispositivo de acceso (PC) y al método empleado para seleccionar el contenido (búsqueda en la web y ejecución por vínculos). No obstante, como confirmaremos más adelante el smartphone es actualmente la piedra angular del consumo online especialmente entre los más jóvenes. Lo que plantea la revisión de estas y otras categorizaciones y los servicios y contenidos que definen. En lo que respecta al término Over-TheTop, podemos ver muchas de sus características reflejadas en las categorías IPVOD, Internet TV e Internet Video planteadas por estos autores.

Más recientemente, la Federal Communications Commission (FCC) estableció una interesante categorización en su informe anual sobre el estado de la competencia en el mercado del vídeo y la televisión, en el que distingue entre plataformas de televisión de pago tradicionales por cable o satélite (multichannel video programming distributors, MVPDs), cadenas tradicionales de televisión por aire (broadcast television stations) y, por último, distribuidores de vídeo online (online video distributors, OVDs). Es en este último grupo es donde clasifica a los operadores Over-The-Top, tanto aquellos que son nativos, como es el caso de Netflix; o bien aquellos otros procedentes de compañías convencionales, como puede ser HBO en el caso de HBO NOW. Sin embargo, los OVDs, tal y como los define la institución norteamericana, constituye una categoría mucho más general para dar cabida también a otros proveedores de contenido audiovisual por Internet, como puede ser Apple a través de iTunes, o Sony por medio de su consola de videojuegos. En base a este informe el área de cobertura de un OVD podría alcanzar todas las regiones capaces de recibir servicios en alta velocidad por Internet. Los consumidores pueden acceder a los contenidos online a través de múltiples dispositivos con conectividad a la red, incluyendo ordenadores, smartphones, tablets, videoconsolas, SmartTVs u otros dispositivos ${ }^{1}$ (FCC, 2017: 620).

Por tanto, la FCC abre el abanico de análisis a otros servicios cuyos modelos de negocio pueden diferir en cierta medida a los planteados por la mayoría de los operadores OTT más generalizados. Es decir, a las fórmulas de suscripción, pago por visión y publicidad, añaden el modelo de negocio típico de iTunes basado en la venta o el alquiler de copias digitales. Este modelo es definido comúnmente como Electronic Sell Through o Transaction Video on Demand (TVOD) (FCC, 2017; European Audiovisual Observatory, 2016). Los consumidores se convierten en propietarios de una copia digital del contenido que podrán almacenar en discos duros locales y/o en servidores remotos (almacenamiento en la nube). Probablemente sea lo más parecido a los negocios editoriales clásicos en su adaptación al entorno digital.

Como bien señalan Álvarez Monzoncillo y López Villanueva (2015: 35) se ha producido una curiosa situación consistente en cierta inversión de los roles habituales de cada actor. Los canales de pago tradicionales, así como los grandes creadores de contenido (HBO o Disney, por ejemplo), están priorizando esfuerzos en la construcción de sus propios canales de distribución online. Sin embargo, las compañías emergentes de distribución por Internet -como es el caso de Netflix, ejemplo paradigmático- dedican cada vez más recursos económicos a la producción de contenido exclusivo y la adquisición de derechos. Aspecto que se confirma al revisar los datos de inversión en producción hechos públicos por Netflix y Amazon, entre otros.

\footnotetext{
1 “(...) an OVD’s geographic service area potentially covers all regions capable of receiving high-speed Internet service. Consumers can access online video via multiple Internet-enabled devices, including computers, smartphones, tablets, gaming consoles, television sets, and other equipment."
} 
Este hecho demuestra una situación lógica e incluso natural: el valor del contenido como reclamo y, en definitiva, como activo que justifica el pago por ciertos servicios; y también la posición esencial que ocupa la distribución en la cadena de valor de las industrias culturales.

Asimismo, los operadores OTT han sido definidos en gran medida según sus propuestas de negocio. El modelo long-tail explica en parte el funcionamiento de estas empresas, basadas en los productos de nicho. Es decir, las OTT explotan un catálogo de contenidos en un mercado masivo, en el cual las compañías encuentran una gran demanda potencial sobre un extensísimo rango de productos especializados (Ojer y Capapé, 2012: 192). En este sentido Kim, Kim y Nam (2016) analizan las relaciones de competencia entre operadores tradicionales y compañías OTT en el mercado coreano según el concepto de la teoría de nichos. Esta teoría explicaría cómo "un organismo compite y coexiste en un entorno dinámico" (2016: 713), buscando su espacio específico donde poder subsistir. Dicho de otro modo, la teoría de nicho ha sido utilizada en múltiples casos para el análisis comparativo entre los nuevos y los viejos medios, y cómo los primeros definen su espacio (su nicho) y se distinguen de entre la competencia en un entorno de coexistencia determinado. De hecho, dentro de la oferta de los OTTs, se pueden hacer distinciones entre OTTs generalistas (como pueden ser Amazon, Netflix o HBO) y OTTs de nicho (como podrían ser los casos de Filmin y FlixOlé).

En definitiva, tras revisar las diferentes implicaciones en torno al concepto OTT, podemos afirmar que, y reduciéndolo al ámbito audiovisual, un OTT es un distribuidor de contenidos audiovisuales que no forma parte del ecosistema tradicional de medios televisivos (ondas hertzianas, cable, satélite e, incluso, IPTV) y que hace uso de la red pública de Internet para llegar a sus clientes, sin el control del operador de red en la transmisión de los contenidos. El acceso se puede realizar a través de cualquier dispositivo conectado, bien sea a través de una aplicación ( $a p p$ ), o bien por medio de la web a través de cualquier navegador.

En todo caso, además de los debates definitorios de lo que es o no es un OTT, importantes para el terreno regulatorio, se hace evidente la creciente preocupación de los medios de comunicación convencionales y de las compañías de telecomunicaciones acerca de las transformaciones que estas nuevas compañías están liderando o pueden liderar en un futuro a corto y medio plazo. Muchas de estas empresas, nacidas bajo el amparo digital, están sustituyendo a los antiguos agentes del sector pero no están sometidos a las mismas obligaciones legales.

\section{Resultados: Análisis de los operadores OTT en un contexto de profundos cambios en el consumo y los ingresos}

El consumo de televisión (en abierto y de pago), así como de contenidos audiovisuales accesibles por otras vías (como la web o las apps) — y en muchos casos no producidos profesionalmente-, ha ido incrementándose hasta alcanzar un récord histórico en el año 2012 con un consumo de 246 minutos por persona y día de televisión lineal ${ }^{2}$. A partir del año 2015, al consumo de televisión lineal, Kantar Media añadió las primeras mediciones de consumo de contenidos en diferido. Lo que significa añadir al consumo de televisión lineal el tiempo dedicado al visionado de contenidos televisivos a través de medios online, o bien a través de algún medio de grabación digital, durante los siete días siguientes al de su emisión convencional. Más recientemente, desde el 1 de marzo de 2017, se incluye además la audiencia de los "invitados" en el hogar. Es decir, contabilizan también a los espectadores que no viven en el hogar en el que se encuentran viendo televisión en ese momento. Dicho de otro modo, aquellos televidentes que no pertenecen a la unidad familiar.

\footnotetext{
${ }^{2}$ Datos publicados por Barlovento Comunicación a partir de información proporcionada por Kantar Media. Disponible en: www.barloventocomunicacion.es [Última consulta el 8 de abril de 2020]
} 
Por tanto, en el año 2017, cuando se inicia la medición de audiencias "invitadas", al consumo de televisión lineal se le suman estas otras dos variables: el consumo en diferido y los espectadores “invitados". De modo que, según los datos de Kantar Media, en 2017, el consumo de televisión ascendió a los 240 minutos por persona y día (223 minutos de televisión lineal, 11 de "invitados" y 5 en diferido). No obstante, lo que también se deduce de estos datos es el progresivo descenso desde 2012 del consumo de televisión lineal o de flujo, exceptuando 2017 cuando se suma por primera vez los minutos correspondientes a televidentes "invitados". En 2019 el consumo de televisión lineal fue de 216 minutos (incluyendo el concepto "invitados" que en 2019 ascendió a 10 minutos), a los que se les suma 7 minutos más de consumo en diferido para un total de 222 minutos.

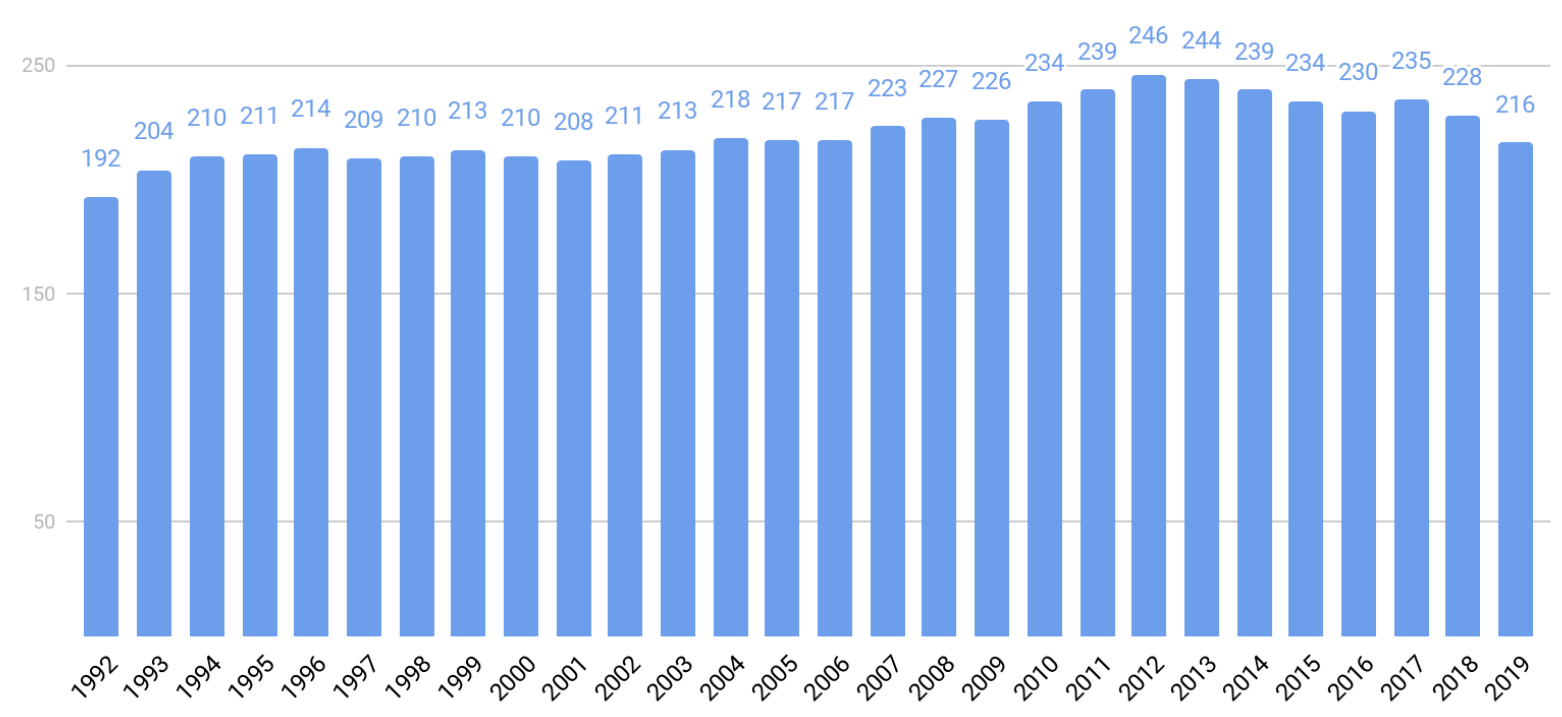

\section{Gráfico 1. Evolución del consumo de televisión lineal (excluyendo el concepto “diferido”). Media de minutos por persona y día.}

Fuente: Elaboración propia / Kantar Media

De modo que, como puede observarse en el gráfico anterior, desde el año 2012 (máximo histórico de consumo televisivo) el visionado de televisión lineal ha perdido 30 minutos hasta los 216 minutos del año 2019. No obstante, para una mejor comparación, si excluimos el valor de los espectadores “invitados" (valor que aún no se contabilizaba en 2012), el año 2019 registra un consumo de televisión lineal de 206 minutos, lo que supondría una reducción de 40 minutos respecto a 2012. Sin embargo, pese a esta nueva variable, la suma de los espectadores "invitados", que sostuvo el consumo de televisión convencional en 2017, la televisión convencional entra en un descenso progresivo que corresponde, entre otros factores, a la consolidación de un contexto de consumo multidispositivo y online; al empuje de la televisión de pago por Internet debido al aumento de las conexiones por fibra óptica (FTTH, Fiber to the Home) y a la definitiva apuesta de empresas OTT por el mercado español, especialmente desde el año 2015.

Es en este segmento donde se están registrando las mayores subidas en cuanto al consumo de contenidos audiovisuales en nuestro país. La televisión de pago, en sus diversas modalidades, casi ha alcanzado los 7 millones de suscriptores en el año 2018 (véase Gráfico 2). 
RLCS, Revista Latina de Comunicación Social, 79, 35-52

\begin{tabular}{ll|l|l|l} 
[Investigación] 10.4185/RLCS-2021-1496 & ISSN 1138-5820 & Año 2021
\end{tabular}

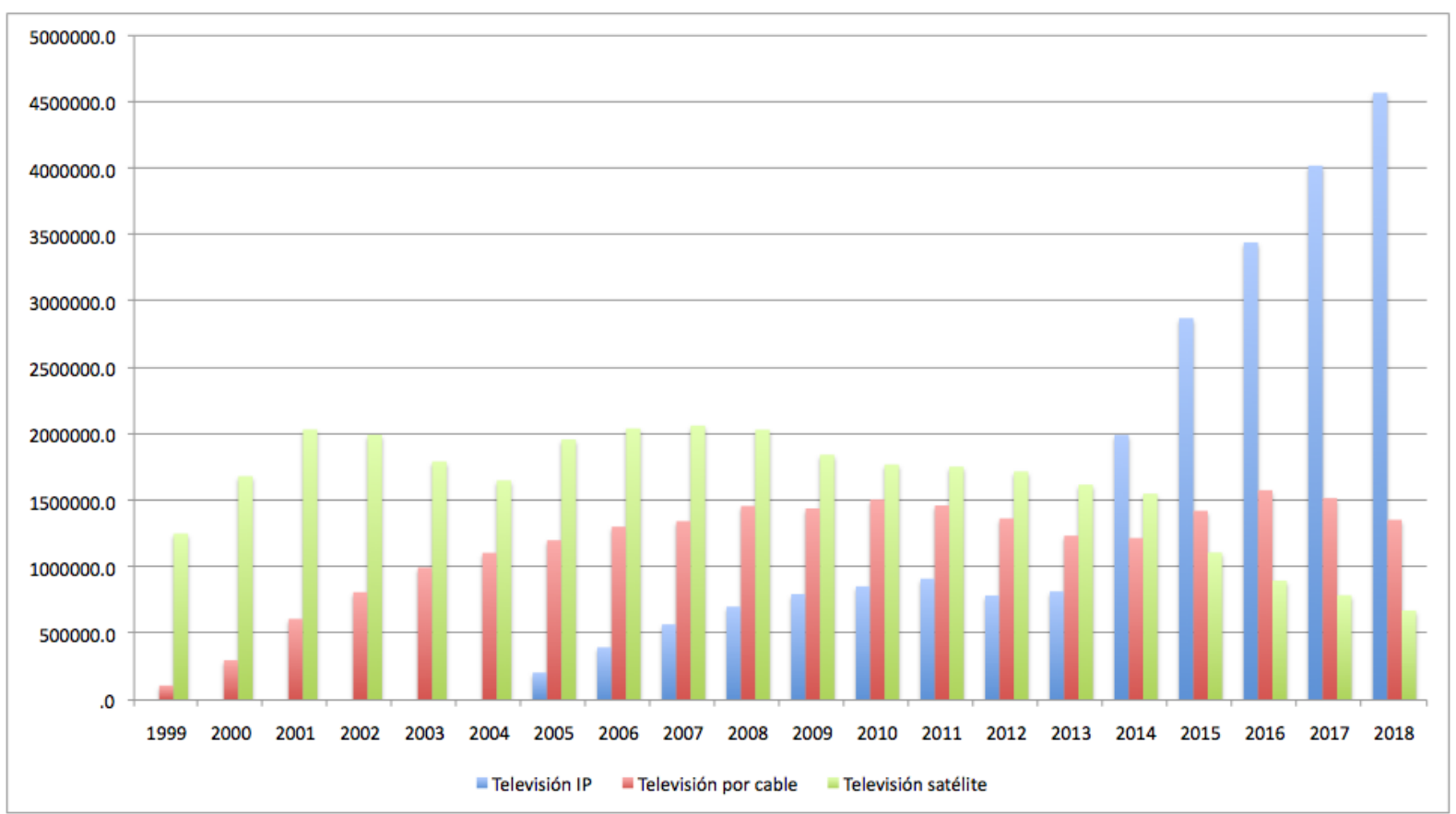

Gráfico 2. Evolución de los suscriptores a televisión de pago por medio de distribución.

Fuente: Elaboración propia / CNMC

Destaca el crecimiento de la IPTV como medio de distribución de servicios de televisión de pago. Desde que arrancaran las primeras plataformas de IPTV en el año 2005, se ha registrado un incremento de 4.363.144 suscriptores en once años (206.572 en 2005 y 4.569.716 en 2016). Actualmente, la IPTV es el medio de distribución de televisión de pago con mayor peso en el mercado español, superando en 3.213.370 abonados a la televisión por cable (1.356.346 en 2018, descendiendo su número de abonados desde 2016 cuando consiguió su mejor dato desde 2005) y en 3.895.739 abonados al satélite (673.977 abonados en 2018).

La televisión de pago por satélite ha experimentado una pérdida de 1.391 .116 abonados respecto al año 2007, cuando alcanzó el máximo de suscripciones desde 1999 (2.065.093 abonados). Esta caída de abonados supone para la televisión de pago por satélite un descenso del $67 \%$ en nueve años: de 2.065 .093 en 2007 a 673977 en 2018, registrando para este último año el mínimo de abonados en casi dos décadas (1999-2018). 
RLCS, Revista Latina de Comunicación Social, 79, 35-52

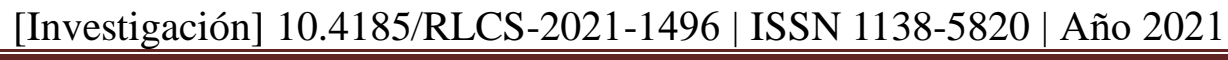

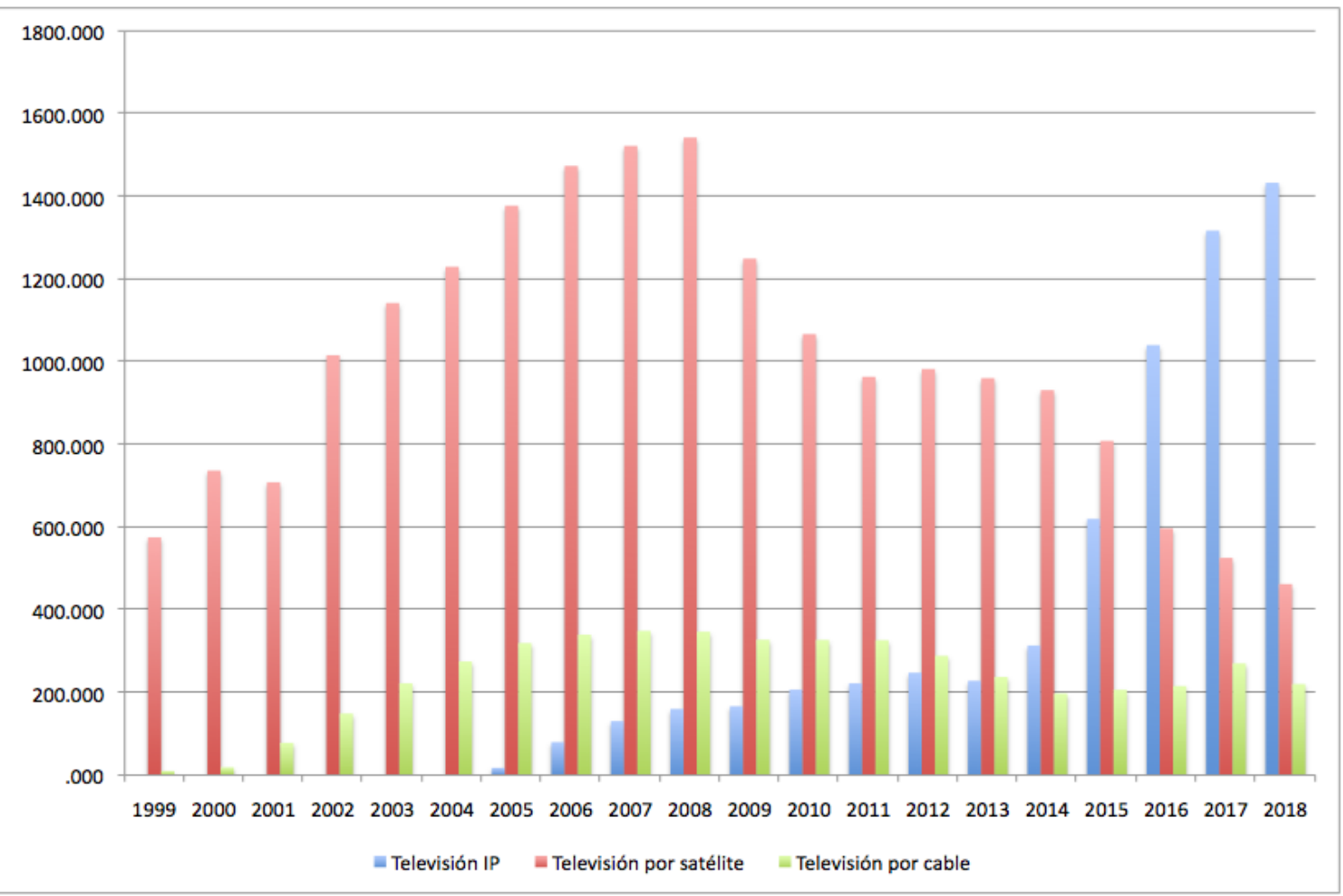

Gráfico 3. Evolución de los ingresos de televisión de pago por medio de distribución. Fuente: Elaboración propia / CNMC

No obstante, atendiendo a los ingresos de la televisión de pago por medio de transmisión o difusión (véase Gráfico 3) el satélite sigue situándose en segunda posición con unos ingresos anuales de 462 millones de euros en 2018 (1080 millones de euros menos, un -70\%, respecto a 2008 cuando alcanzó su máximo histórico). Tras el satélite, el cable registra 220,2 millones de euros de ingresos en 2018. La televisión IP, por el contrario, incrementa sus ingresos en 1.119 millones de euros en tan sólo 4 años (de 2014 a 2018).

Los datos obtenidos por las plataformas de IPTV se explican, entre otras razones, por la manera en que las compañías de telecomunicaciones han comercializado sus servicios mediante paquetes. En la actualidad, es el five play o paquete quíntuple el que está registrando mayores subidas, incluyendo televisión a los servicios habituales de telefonía (fija y móvil) y conexión a Internet (en el hogar y el teléfono móvil). De hecho, 9 de cada 10 suscriptores contrataron la televisión de pago por medio de algún paquete de servicios, según la CNMC. De este modo, los servicios "empaquetados" están permitiendo cierta retención de suscriptores a servicios de televisión de pago basados en la tradicional oferta de múltiples canales de emisión lineal. Según una reciente encuesta de la consultora Deloitte (Deloitte Digital Media Trends) los suscriptores a estos servicios, sea cual sea la tecnología empleada, cada vez pierden más interés ante las plataformas que ofrecen cientos de canales. Lo que en otra época supuso un excitante acceso a miles de horas de entretenimiento tematizado, si bien sujetos al flujo de la programación, ahora son vistos como servicios innecesarios, inflados y frustrantes (Dreier, 2018).

El siguiente gráfico muestra una comparativa de los ingresos de la televisión en abierto y de pago, en el que se observa cómo desde 2016 la televisión de pago obtiene mayores ingresos que la televisión en abierto (2.162,11 millones de euros y 1.797,51 millones de euros en 2018, respectivamente). 
RLCS, Revista Latina de Comunicación Social, 79, 35-52

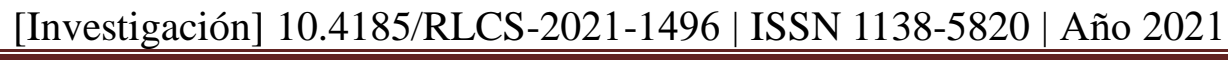

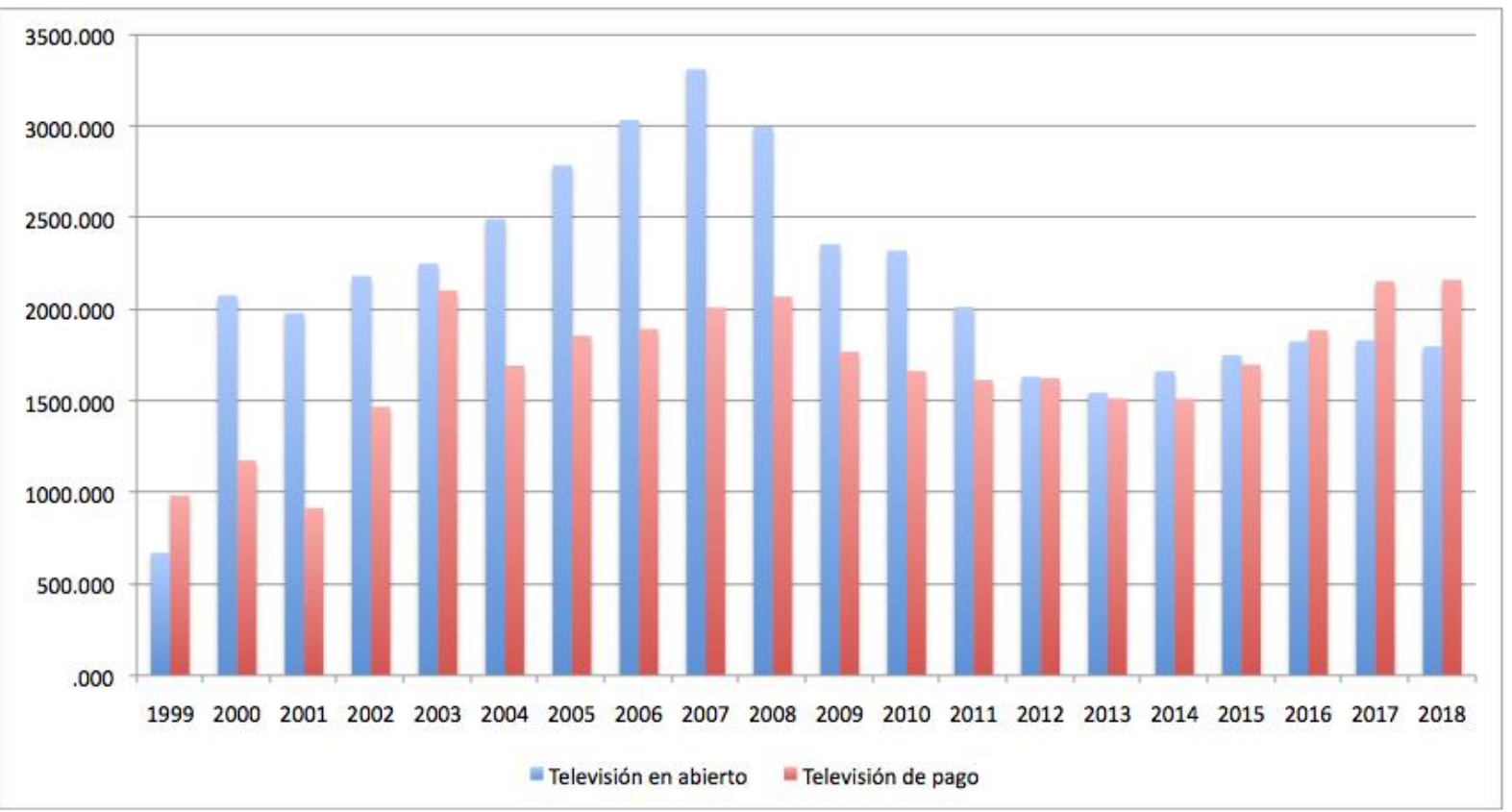

Gráfico 4. Comparativa entre los ingresos de televisión en abierto y de pago (sin subvenciones). Expresado en millones de euros.

Fuente: Elaboración propia / CNMC

Como se deduce de los resultados ofrecidos por la CNMC, exceptuando los años 1999 y 2003, nunca existió tal igualdad desde el año 2012. Mientras que la televisión en abierto se encuentra lejos de los ingresos obtenidos durante el periodo de 2005 a 2008, la televisión de pago sí que ha conseguido recuperar parte del terreno perdido alcanzando cotas, e incluso superándolas, a los años previos de la crisis económica iniciada en 2008. Esta recuperación se debe en parte a que el descenso en sus ingresos a partir de 2008 no ha sido tan acusado como en la televisión en abierto.

En el año 2018 la televisión en abierto obtuvo unos ingresos por valor de 1.797,51 millones de euros, según la CNMC. Lo que significa una reducción de 1.515 millones de euros respecto a 2007 (un descenso del 45,7\%), año en el que se consiguió el tope de 3.312,54 millones. Por su parte, la televisión de pago ha conseguido unos ingresos de 2.162,11 millones durante el año 2018; lo que supone un aumento de 58,51 millones en comparación con 2003, cuando consiguió su máximo histórico (2.103,6 millones).

Desde 2014 la televisión de pago ha conseguido incrementar sus ingresos en un 30\% (de 1.511,95 millones en 2014 a 2.162,11 millones en 2018). La televisión en abierto, en cambio, consiguió incrementar sus ingresos en un 10\% hasta 2017. Sin embargo, en 2018 y 2019 (como vimos en la introducción de este trabajo) los ingresos de la televisión en abierto vuelven a descender.

Respecto al número de suscriptores de compañías OTT, la CNMC dejó de publicar datos oficiales, por lo que desde 2019 carecemos de información al respecto debido a la dificultad de conseguir cifras fidedignas por este organismo. Esto se debe a las estructuras corporativas de estas compañías tecnológicas que entroncan con la problemática acerca de su régimen fiscal y sus obligaciones de transparencia. Empresas como Netflix o Disney+ facturan a sus clientes españoles desde matrices ubicadas en Amsterdan (The Walt Disney Company BV y Netflix International BV) lo que les permite "esquivar" impuestos y otras obligaciones (tasas para financiación de la televisión pública y producción cinematográfica), así como la obligación de informar sobre el número de clientes o suscriptores a organismos como la CNMC (Martínez, 2020). 


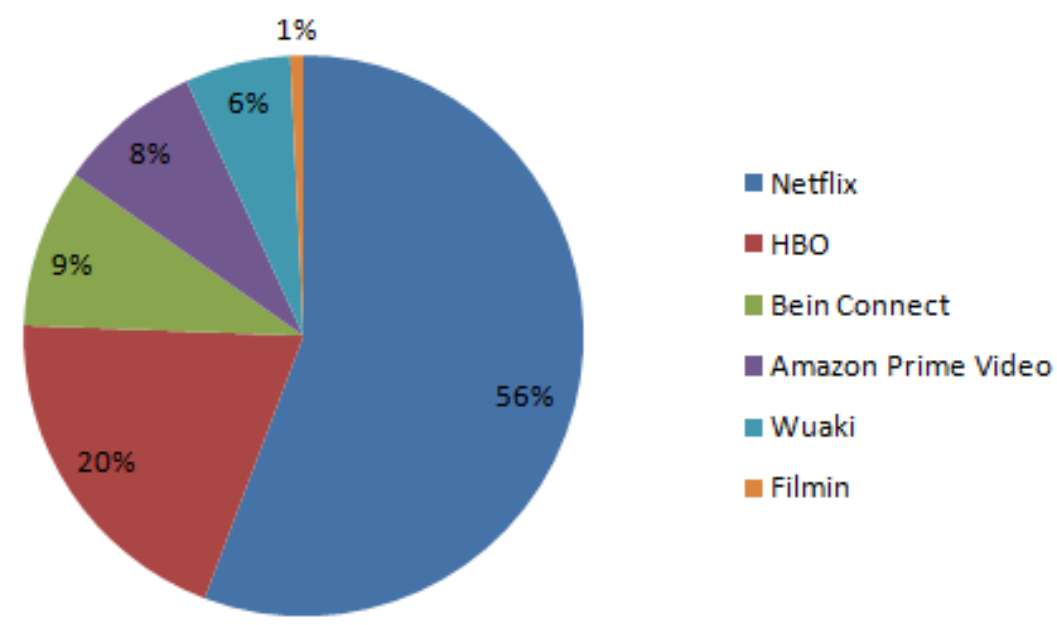

Gráfico 5. Porcentajes por OTT sobre el total de suscriptores (excluyendo operadores de telecomunicaciones).

Fuente: Elaboración propia / CNMC

Datos correspondientes a la primera mitad del año 2017 situaba como compañía líder en España a Netflix con 1.163.000 suscriptores. Le siguió HBO con 414.000 abonados en su primer año de actividad. En tercer lugar se encontraba Bein Connect (plataforma propiedad de Mediapro y que integró en 2014 los servicios de Total Channel) con 191.000 suscriptores. No obstante, Bein Connect cesó su actividad en nuestro país marzo de 2020. Por su parte, Amazon Prime Video y Rakuten Wuaki acumulaban 175.000 y 127.000 suscriptores, respectivamente. Y, por último, la plataforma de cine independiente Filmin registró 16.000 suscripciones.

Es decir, Netflix concentraba el $56 \%$ del total de suscripciones o cuentas a servicios proporcionados por OTTs (véase Gráfico 5). Sólo entre Netflix y HBO concentraron cerca del 80\% de las suscripciones en España.

Al cierre del primer semestre de 2018, según la CNMC (correspondiente a los últimos datos aportados por la comisión) Netflix superó a los dos millones de hogares suscritos, seguido por Amazon Prime Video 656.000 suscriptores y HBO con 475.000 cuentas. Asimismo, Rakuten Wuaki registró 147.000 suscripciones, Bein Connect 377.000 y Sky 114.000. Esta última plataforma, propiedad de News Corp, se lanzó en nuestro país en septiembre de 2017 (El Español, 2017) y viene a definir una variante de OTT llamada skinny bundles y designa a aquellos servicios que distribuyen paquetes de contenidos audiovisuales que ofrecen menos canales de pago que los que tradicionalmente se ofertan, a precios más reducidos.

Aún no existen datos sobre las últimas compañías en integrarse a la surtida oferta de contenidos por internet en el mercado español: Disney+, Apple TV+ o la española FlixOlé, lanzada por Mercury Films. La compañía que dirige Enrique Cerezo se apoya en tecnología desarrollada por la empresa sueca Magine que ya en 2013 intentó lanzar su propio servicio en España (bajo la fórmula skinny bundles) sin mucho éxito, cesando sus actividades en nuestro mercado a principios de 2015 (Panorama Audiovisual, 2015). 


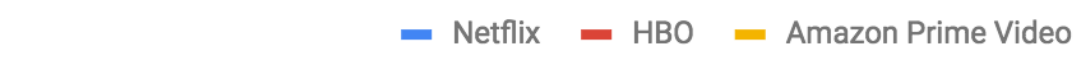

2.500 .000

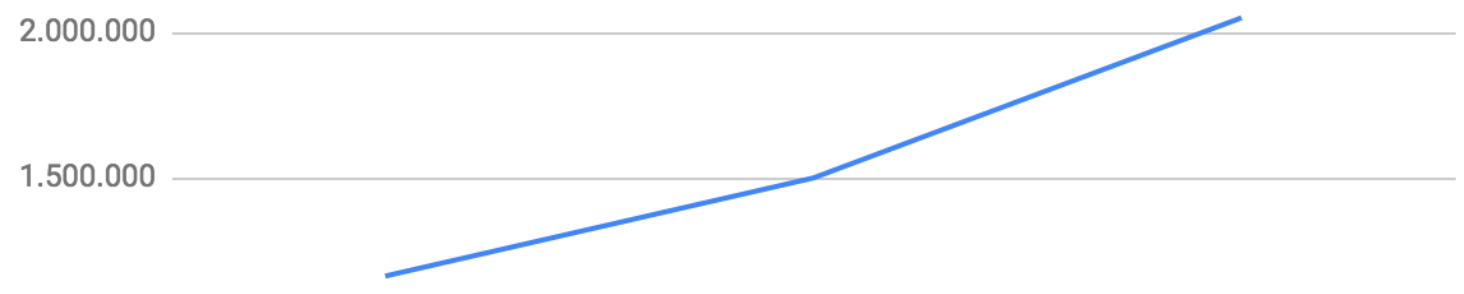

1.000 .000

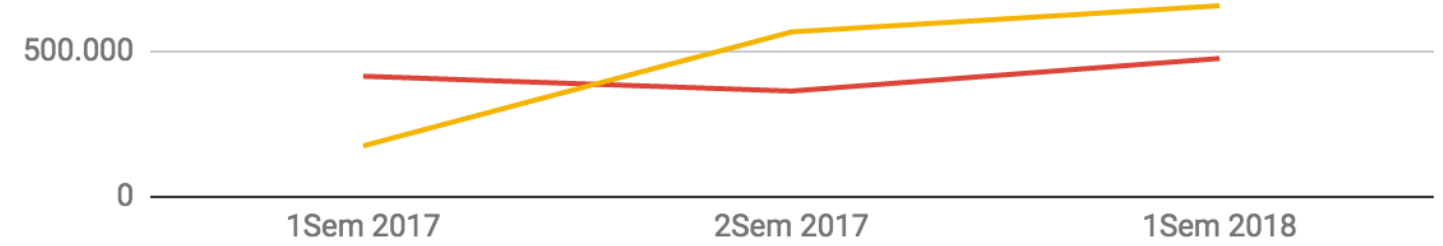

Gráfico 6. Evolución en número de suscriptores de las principales OTT (medido en semestres hasta junio 2018).

Fuente: Elaboración propia / CNMC

Durante esas mismas fechas se dieron a conocer las intenciones de AMC Networks España de ofrecer un servicio bajo demanda (bautizado como "Selekt") con la inclusión de contenidos de 10 canales de televisión lineal. No se conocía exactamente la fórmula a seguir por AMC hasta que, finalmente, se confirmó que dicho servicio de contenidos bajo demanda se integraría como servicio adicional para las compañías de telecomunicaciones. Orange publicó el 31 de marzo de 2020 que AMC Selekt se integraría en su servicio de televisión sin coste adicional a partir de esa fecha ${ }^{3}$.

También Huawei, en alianza con Atresmedia como proveedor de contenidos (Muñoz, 2017), anunció a finales de 2017 su intención de entrar en el negocio con Huawei Video durante el primer trimestre de 2018. Según las informaciones publicadas en prensa a raíz de este anuncio, el objetivo de la compañía china es la de lanzar el servicio primero en España e Italia, para más tarde hacerlo extensible a otros mercados europeos. El acuerdo alcanzado con el grupo Atresmedia es la señal de su apuesta por el contenido de carácter local, combinado con producciones internacionales.

En el siguiente gráfico, de acuerdo al último panel de hogares realizado por la CNMC, en el que recogen datos para el primer semestre de 2018, se puede observar la evolución en el porcentaje de uso de servicios OTT para el total de hogares con acceso a internet en España.

\footnotetext{
3 “AMC Selekt, el servicio bajo demanda más variado en exclusiva en Orange TV", publicado el 31 de marzo de 2020. Disponible en: https://poptv.orange.es/en-orange/amc-selekt [Consultado el 10 de abril de 2020].
} 
RLCS, Revista Latina de Comunicación Social, 79, 35-52

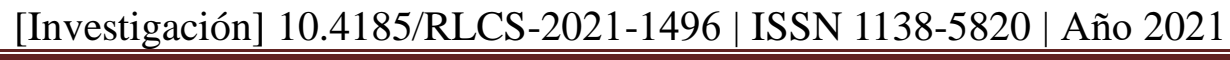

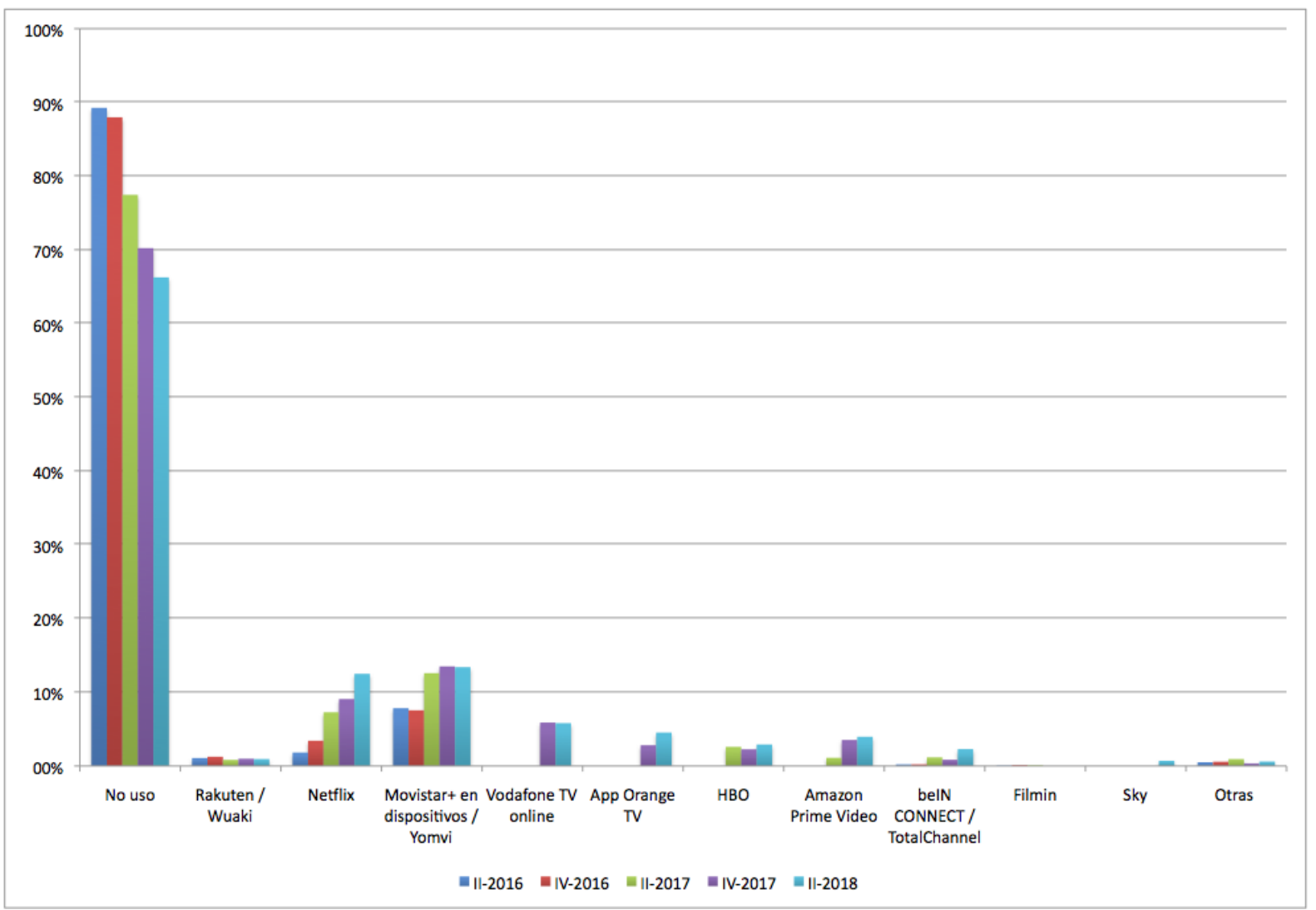

Gráfico 7. Evolución en el uso de servicios OTT de pago (porcentaje de hogares). Datos para el segundo trimestre de 2019.

Fuente: Elaboración propia / CNMC

Se hace evidente, pues, a la luz de los datos observados, que el porcentaje de hogares que declaran no hacer uso de estos servicios aún resulta significativamente alto si bien se ha visto reducido en un $23 \%$ desde 2016 hasta el primer semestre de 2018. Lo cual además puede ser visto como muestra del potencial del mercado de servicios online por streaming. Entre todos ellos, Movistar+ (antiguo Yomvi) se sitúa en cabeza con un 13,4\% (un 5,6\% más) hasta el primer semestre de 2018 gracias a la amplia base de clientes de sus servicios de pago para IPTV.

Por tanto, de entre las compañías independientes respecto de operadoras de telecomunicaciones, destaca como cabía esperar el impulso de Netflix en el mercado español con un crecimiento del 10,7\%, durante el mismo período de tiempo, en cuanto a hogares que utilizan Netflix para ver contenidos audiovisuales de pago por internet. De modo que se hace evidente el impulso en la oferta audiovisual de este segmento en el último año con una amplia variedad de jugadores que ha seguido creciendo en los últimos meses. Las incógnitas surgen entorno a sus modelos de negocio y formas de diferenciación respecto a la competencia, así como la viabilidad en el número de actores y la percepción y usos de los internautas.

Asimismo, resulta significativo incluir en este análisis las estimaciones sobre los presupuestos que compañías como Netflix dedican a la producción de contenidos originales, que supone la gran baza de estas compañías para atraer y mantener suscriptores (con casos excepcionales como el de Friends, cuya cotización sigue al alza tras 30 años desde su estreno). Según datos recientes, Netflix gastó en 2019 más de 14.500 millones de dólares, un incremento de casi cinco mil millones respecto a 2017. 


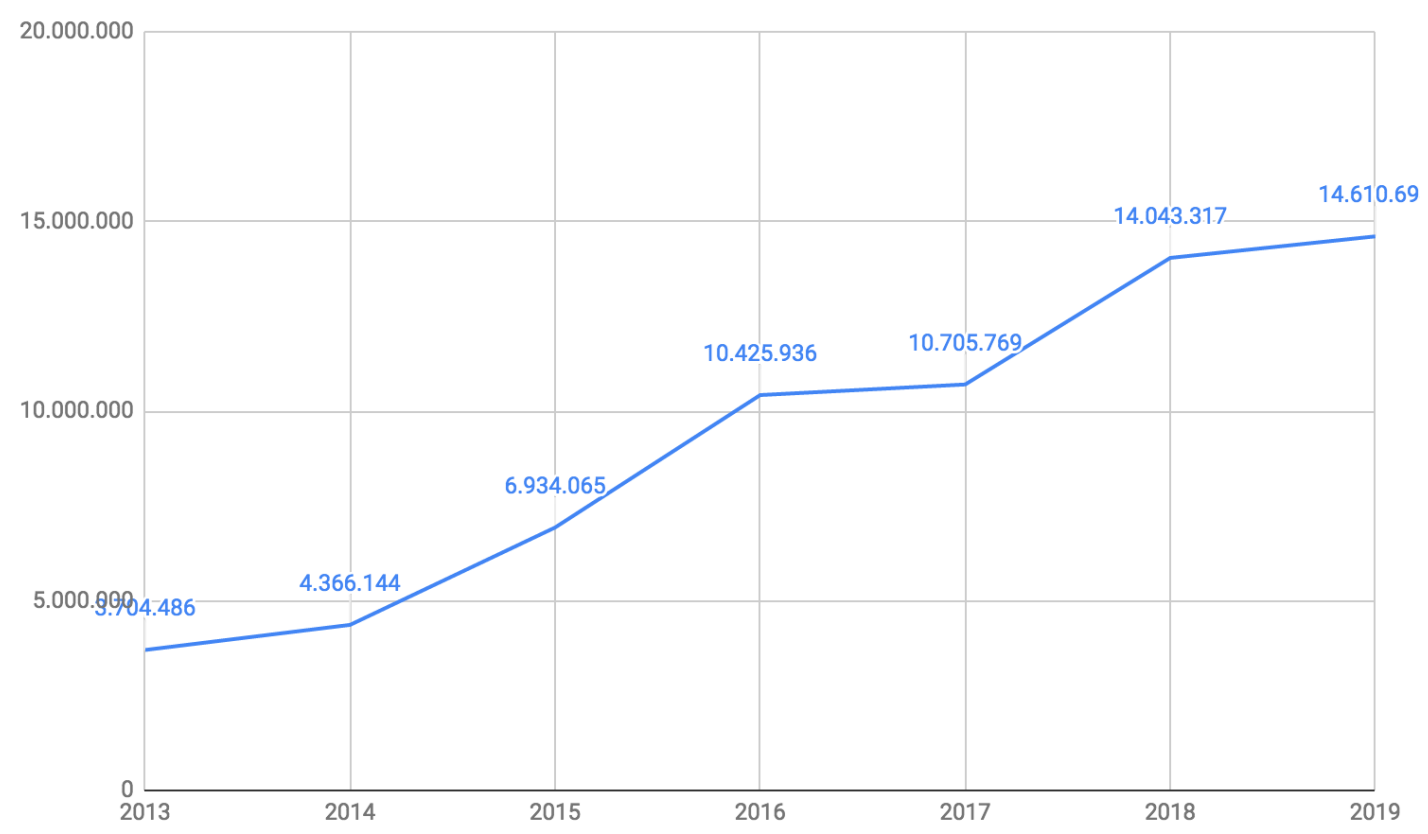

Gráfico 8. Inversión de Netflix en contenido original (en miles).

Fuente: Elaboración propia / Netflix Investors

En base a los datos que Netflix hace públicos para sus accionistas, la compañía de Los Gatos ha incrementado el gasto en producción original en casi 11 mil millones de dólares en 7 años. Su apuesta por este tipo de contenidos conlleva un gasto prácticamente exponencial, si bien el gasto entre 2018 y 2019 es prácticamente el mismo (un incremento de algo más de medio millón).

Igualmente es destacable el importante crecimiento de la televisión de pago respecto a los ingresos publicitarios en sus diferentes medios de transmisión. Según datos del último informe de Infoadex, los canales de pago de televisión han visto crecer sus ingresos por publicidad hasta algo más de 100 millones de euros. En cuanto a plataformas de pago, Movistar+ lidera el camino en los ingresos gracias a su agresiva campaña de concentración de contenidos. Especialmente los relativos al deporte, ámbito en el que ha adquirido derechos de retransmisión sobre las competiciones más atractivas para el público (fútbol, Fórmula 1, MotoGP, Euroliga y liga ACB de baloncesto). Además, Movistar+ se suma a la acción clave de todas estas compañías: la seria apuesta por la producción de contenido de ficción exclusivo. Un ejemplo de ello es el estreno el 8 de enero de 2018 de la serie La Peste, sin duda uno de los mejores estrenos de la plataforma para una serie de ficción, superando en aquellos entonces a grandes superproducciones internacionales como Juego de Tronos (antes de su desenlace) o The Walking Dead.

La décima oleada del informe Televidente 2.0, elaborado por Telefónica, Ymedia y The Cocktail Analysis ${ }^{4}$, proporciona interesante información acerca de la evolución tecnológica y su incidencia en los cambios del consumo de contenidos audiovisuales. Su metodología, basada en grupos de discusión y encuestas, está dirigida a una población internauta general comprendida entre los 18 y los 55 años.

El informe revela una importante caída del consumo de contenido lineal entre la muestra objeto de estudio, especialmente en TDT pero también entre la oferta de canales convencionales que ofrece la

\begin{tabular}{lcccccc}
\hline 4 & Informe & Televidente & 2.0 & $2016-2017$ & $(X$ & Oleada $),$
\end{tabular}


IPTV. Pese a todo, su peso dentro del consumo audiovisual sigue siendo muy importante, constituyendo prácticamente la mitad de las visualizaciones de los internautas participantes del estudio (30\% en el caso de la TDT y $23 \%$ para canales en servicios IPTV). La otra mitad de las visualizaciones de los internautas se divide en diferentes formas de consumo bajo demanda (no lineal o en diferido, siguiendo la definición dada por Barlovento Comunicación), como puede ser el catch-up, las apps, la web o algún sistema de grabación (en la nube o en discos duros locales).

Según este informe, el $51 \%$ de los internautas tiene contratado algún servicio de televisión de pago (OTT o IPTV) de los que el 16\% están suscritos a ambos servicios, el 9\% son clientes exclusivos de operadores OTT y el $26 \%$ clientes exclusivos de IPTV.

\section{Conclusiones}

A la luz de los datos analizados se confirma la tendencia de cambio hacia una forma de consumo audiovisual más desestructurado y hasta en cierta medida más compulsivo, especialmente entre los más jóvenes. El satélite ha perdido gran parte de su peso en el mercado como vehículo de transmisión de servicios de televisión de pago en beneficio de internet, que se consolida como el sistema de mayor importancia.

Aunque la incidencia de estos cambios en la industria se hace evidente en los datos económicos y de consumo de las plataformas tradicionales (televisión lineal), realmente es en el sector del pago y sus servicios online donde se están desarrollando los cambios de mayor envergadura. De hecho, la televisión convencional mantiene cierta tendencia positiva en minutos de consumo por persona y día en aquellos públicos de mayor edad. Todavía más en los últimos años al añadir las mediciones de las audiencias "invitadas", un valor que incrementa el consumo y considera a un público invisible hasta la fecha para el mercado publicitario.

Sin embargo, las diferentes vertientes de los servicios de pago a través de internet han igualado su peso en la industria española respecto a los operadores tradicionales. Por tanto, se puede afirmar que las transformaciones de la industria se circunscriben en su mayoría al terreno online. Haciendo especial hincapié en las compañías de telecomunicaciones como motores de desarrollo de la televisión de pago. Una modalidad basada en la comercialización dentro de los paquetes de servicios de comunicación y entretenimiento, lo que en muchos casos deriva en la degradación del valor percibido de estos servicios televisivos.

Las OTT y su modelo de negocio basado en el long-tail compensan los ajustados precios de sus suscripciones con una explotación intensiva de las economías de escala y por la apuesta, cada vez más importante, de un modelo mixto en el que la publicidad sirve de complemento gracias a un uso inteligente del emplazamiento de producto. De hecho, en el último año las inversiones publicitarias en OTT crecieron algo más de un 30\%, si bien aún las cifras absolutas representan un valor marginal de sus ingresos totales basado en un modelo fundamentalmente de suscripción.

Autores como Álvarez Monzoncillo y López Villanueva (2015: 36) destacaban que una de las posibles claves para el crecimiento de las OTT residía en su capacidad por conseguir derechos sobre contenidos que ya estaban en posesión de otros operadores convencionales como Movistar+, por ejemplo. Sin embargo, estas compañías se han hecho fuertes en la creación de contenido original y en la consolidación de un ecosistema de consumo flexible y adaptable. Lo cual supone su principal ventaja y elemento diferenciador respecto a las fórmulas convencionales de televisión de pago. Su penetración entre los más jóvenes está siendo fulgurante, lo que también pondrá en discusión el papel de la televisión en abierto lineal. 
Por otro lado, algunas vías que podrían permitir la viabilidad de estos modelos pasaría por replicar las estrategias televisivas más convencionales, como la distinción entre canales generalistas y temáticos. Una tendencia plausible puede pasar por la puesta en marcha de OTTs centrados en una temática o público y así explotar los múltiples y diversos nichos en base al modelo long tail. Es una tendencia que podría comercializarse por paquetes, tal y como hacen las compañías de telecomunicaciones para sus servicios de telefonía, internet y televisión.

Así pues, se confirma la hipótesis planteada al inicio al encontrar un panorama audiovisual sobreabundante que está transformando las formas de consumo pero también la percepción y las expectativas del público en torno al entretenimiento audiovisual. Lo cual supone un beneficio para los espectadores respecto a la percepción general de que las producciones para televisión están obteniendo un incremento de calidad muy significativo, especialmente aquellas relacionadas con la ficción. Pero esta saturación conlleva también un problema de visibilidad de los contenidos y un problema de agotamiento para los espectadores. Además, el incremento de la competencia tiende a incrementar, a su vez, los costes de producción. Los operadores OTT, por el momento, se encuentran más centrados en la creación y adquisición de contenido original, sin embargo, no es descartable que en un futuro a corto plazo puedan entrar a competir por los derechos de ciertas retransmisiones de gran demanda como son el fútbol (como pretende Dazn al apostar exclusivamente por el deporte), los grandes eventos y las películas de éxito internacional. Lo que derivaría en un serio perjuicio para los servicios de televisión convencionales.

Por todo lo expuesto se prevé un estancamiento de la producción para todas las plataformas y un reordenamiento del panorama, que llevará a la consolidación de los modelos bajo demanda y a previsibles movimientos empresariales dirigidos a la concentración.

\section{Referencias}

Álvarez-Monzoncillo, J. M. y López-Villanueva, J. (2015). Vidas paralelas de las películas: circuitos estratificados de distribución y consumo. AdComunica. Revista Científica de Estrategias, Tendencias e Innovación en Comunicación, 10, 21-40. https://doi.org/10.6035/21740992.2015.10.3.

Barlovento Comunicación (2020). Análisis Audiencias TV: Marzo 2020. https://bit.ly/2VohkBC

Barlovento Comunicación (2019). Análisis Televisivo 2019. https://bit.ly/3a6Ho9A

El Español (2017, 31 de agosto). Sky confirma en redes sociales su desembarco en España. El Español. https://bit.ly/3a2NAzt

Dreier, T. (2018, 20 de marzo). Deloitte Sees Value Gap Between What Pay TV Viewers Want and Get. Streaming Media. https://bit.ly/34wJv5B

European Audiovisual Observatory (2016). VOD, platforms and OTT which promotion obligations for European works? IRIS plus 2016-3. https://bit.ly/2yc4huR

FCC (2017). Annual Assessment of the Status of Competition in the Market for the Delivery of Video Programming. https://bit.ly/3a4HKO7 
García-Santamaría, J. V., Pérez-Serrano, M. J. y Alcolea-Díaz, G. (2014). Las nuevas plataformas televisivas en España y su influencia en el mercado. Revista Latina de Comunicación Social, 69, 390-417. https://doi.org/10.4185/RLCS-2014-1017

InfoAdex (2020). Estudio InfoAdex de la inversión publicitaria en España 2020. https://bit.ly/2xfa6Id

Kim, J., Kim, S. y Nam, C. (2016). Competitive dynamics in the Korean video platform market: Traditional pay TV platforms vs. OTT platforms. Telematics And Informatics, 33(2), 711-721. https://doi.org/10.1016/j.tele.2015.06.014

Marcos, N. (2016, 7 de junio). De la edad de oro de la televisión a ¿la sobredosis? El País. https://bit.ly/2JVVxfh

Martínez, J. (2020, 25 de marzo). Disney+ se suma a Netflix y facturará a los clientes españoles desde Países Bajos. La Información. https://bit.ly/2wyEsoB

Muñoz, R. (2017, 27 de octubre). Huawei se une a Atresmedia para crear el Netflix español. El País. https://bit.ly/3edeDeB

Newsline Report (2017, 9 de agosto). Disney abandona Netflix y lanza plataforma propia. https://bit.ly/2RwSSwS

Ojer, T. y Capapé, E. (2012). Nuevos modelos de negocio en la distribución de contenidos audiovisuales: el caso de Netflix. Revista Comunicación, 1(10), 187-200. https://bit.ly/3edOjB7

Panorama Audiovisual (2015, 13 de enero). Magine TV abandona el mercado español. https://bit.ly/2y5itGi

Scarpellini, P. (2018, 9 de marzo). Jon Favreau, el último fichaje del universo Star Wars. El Mundo. https://bit.ly/3ehKB9N

Simpson, W. y Greenfield, H. (2009). IPTV and Internet Video: Expanding the Reach of Television Broadcasting. Elsevier.

\section{AUTOR}

\section{José Borja Arjona Martín.}

Profesor Titular de Comunicación Audiovisual de la Universidad de Granada (España). Sus principales líneas de investigación se centran en la televisión interactiva, el vídeo en Internet, la comunicación viral y las nuevas formas de producción y entrega como crowdsourcing.

Orcid ID: https://orcid.org/0000-0003-3094-0020

Google Scholar: https://scholar.google.com/citations?hl=es\&user=W94uXAoAAAAJ 